\title{
Early Ontogenetic Stages in Dolipore/Parenthesome Formation in Polyporus biennis
}

\author{
By R. T. MOORE \\ School of Biological and Environmental Studies, The New University of Ulster, \\ Coleraine, Northern Ireland
}

(Received 28 August 1974; revised 2I October 1974)

SUMMARY

Sheared hyphae of Polyporus biennis, allowed to grow for I $5 \mathrm{~h}$, showed a number of stages of dolipore/parenthesome formation. These included: the proliferation of new endoplasmic reticulum from the nuclear envelope; the differentiation of endoplasmic reticulum into parenthesomes that initially were conjoined and free in the cytoplasm; the immature appearance of the electron-transparent dolipore shell; and the development of the apertural kernel into the occlusions at the dolipore orifices.

\section{INTRODUCTION}

There is now an extensive literature on the ultrastructure of basidiomycete septa from representatives of most of the major taxa. In one of the most recent studies, Moore \& Marchant (1972) critically characterized the components of the dolipore/parenthesome septum of Polyporus biennis. In their discussion and model of this complex they emphasized the seemingly crystalline structure of the parenthesome, its continuity with the endoplasmic reticulum of the septum as well as with the principal components of the dolipore, cross-wall layers continuous into the inflated portion, a light shell lining the dolipore, and the champagne-cork configuration of the pair of inserted occlusions. Their observations, as well as most of the others in the literature, were of mature septa. There are few studies purporting to show stages of septum formation (Girbardt, I96I ; Bracker \& Butler, 1963; Jersild, Mishkin \& Niederpruem, 1967; Raudaskoski, I970). I report observations on the ultrastructure of ontogenetic stages in the formation of dolipore/parenthesome septa of Polyporus biennis.

\section{METHODS}

A dikaryotic strain of Polyporus biennis (Bull. ex Fr.) Fries obtained from the Centraalbureau voor Schimmelcultures, Baarn, The Netherlands (CBS676.70) was grown on potato dextrose agar. Colonies about a week old were sheared in a blender and samples were pipetted into Petri dishes containing potato dextrose medium. The regenerating hyphae were allowed to grow for $\mathrm{I} 5 \mathrm{~h}$ and then centrifuged and prepared for electron microscopy. The concentrated hyphal pellets were fixed in $\mathrm{I} \%$ aqueous potassium permanganate for Io min at room temperature, dehydrated in an acetone series interrupted by staining overnight in $\mathrm{r} \%$ uranyl nitrate in $70 \%$ acetone, embedded in Epon 8I 2, and sectioned with a diamond knife on either an LKB Ultratome or a Huxley Ultra-microtome (Cambridge). Sections on uncoated grids were examined in either a Philips EM 300 or an AEI 6G electron microscope at $80 \mathrm{kV}$. 
R. T. MOORE
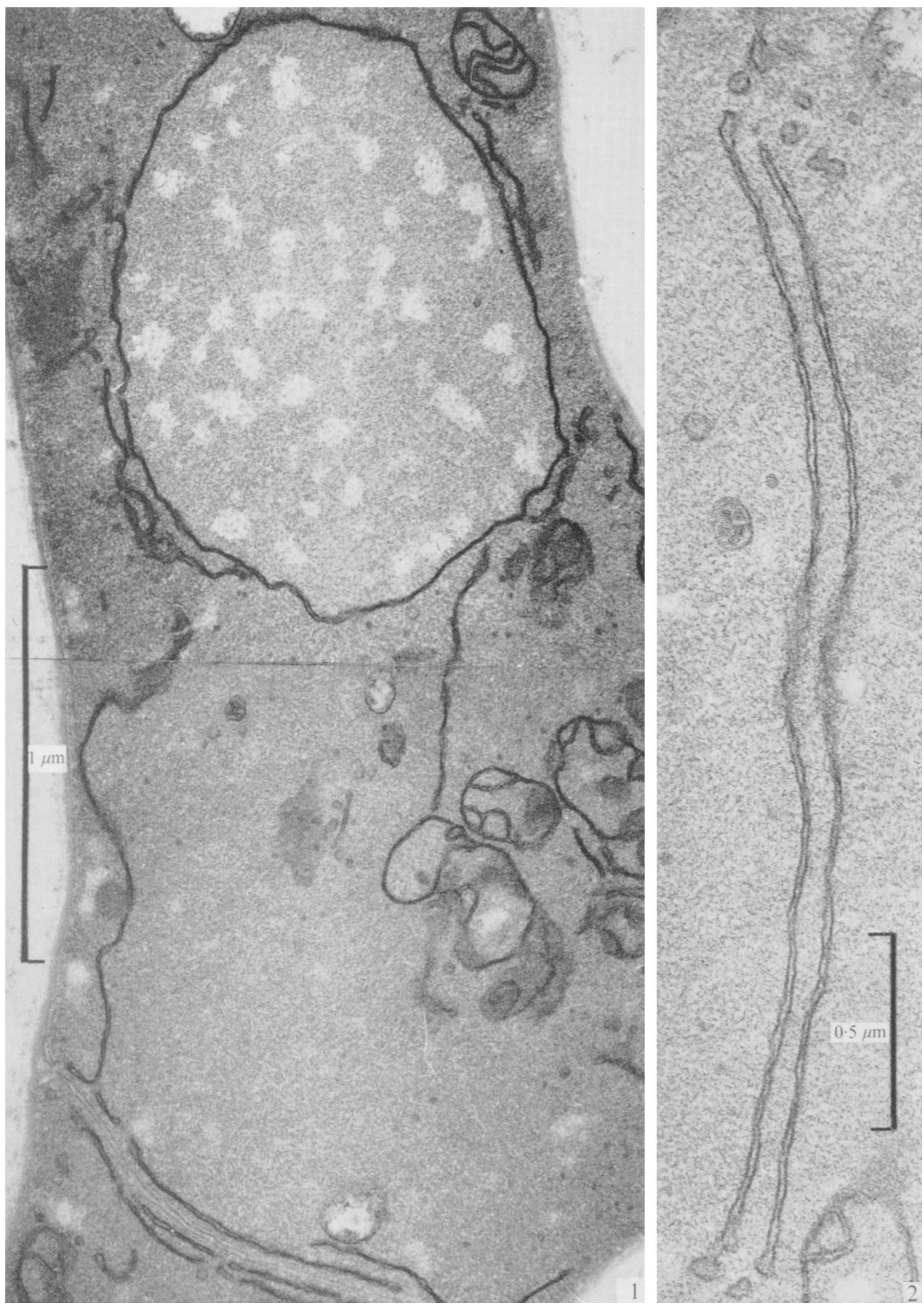


\section{RESULTS}

In the prepared material of $P$. biennis there occurred a diversity of profiles ranging from mature septa to configurations that seemed reasonable antecedents of this condition. One of the peculiarities of these cells was the distribution of endoplasmic reticulum (e.r.). It was prominent along the septa, including that differentiated into the parenthesomes, but was sparse in the cytoplasm and virtually lacking along the hyphal walls. The formation of new septa and associated parenthesomes, therefore, probably required a supply of new e.r. One possible source of new membranes is the nuclear envelope. This is the interpretation given to Fig. I, in which there is e.r. that is continuous with the nuclear envelope, that occupies the cytoplasm between the nucleus and the septum, and that is continuous with e.r. along the septum. An example of the continuity of cytoplasmic e.r. with that of a mature parenthesome is given in Fig. 6.

Parenthesomes appear to be differentiated from cytoplasmic e.r. An early developmental stage is the apparent parallel orientation of e.r. elements and their differentiation of pairs of parenthesomes before septum formation (Figs. 2 to 6). Figure 2 shows a pair of parallel e.r. elements in median section, as evidenced by the sharp profile across most of their length.

In the centre of this configuration there is a blurring, possibly suggesting a change occurring. The parallel array of e.r. in Fig. 3 shows a central region that had become fenestrated. Parenthesome sections were frequently found free in the cytoplasm and variously continuous with e.r. elements (Figs. 4 to 6 ). Figures $4(a)$ and $(b)$ are serial sections and it is evident that the paired parenthesomes were partially conjoined; the typical bilamellate condition is discernible in Figs. $4(b)$ and 5 (compare with Fig. 10). Figure 5 shows two parenthesome elements, one with an e.r. continuity. Figure 6 presents two parenthesome fragments in tangential section. Both are clearly continuous, with undifferentiated e.r., and both show characteristic pores. Mature parenthesomes (Figs. 7 to I0), found associated with dolipore septa, were perforate, marginally continuous with septal e.r., and optically bilamellate with the outer face exhibiting a greater electron translucency than the inner.

Stages in dolipore formation are shown in Figs. 8 to 10. Young septa were strongly demarcated by adjacent e.r. on both sides (Fig. 8) and were composed of three layers (Figs. 9 and I0) : a middle light layer that adjoined the hyphal wall was bounded on either side by dark layers that merged with the inner portion of the hyphal wall (Figs. 7 and 9). Sections of the developing dolipore (Fig. 8) show three plaques of light material beneath the plasma membrane on each of the opposing lips, two on the back shoulder and one on the pore-channel face. The pore channel itself contained a granular material, and the granular occluding material had become darker and was either limited to the pore channel as a kernel with a figure-eight profile (Fig. 9) or appeared as small domes outside the pore orifices (Fig. I0).

Fig. I. Longitudinal section of part of a hypha, showing one of the two nuclei and part of a septum. Of particular significance is the distribution of e.r. (endoplasmic reticulum): it does not lie along the walls but lines both sides of the septum, occupies the cytoplasm between nucleus and septum (one element being continuous with the septal e.r.), and has several elements in the paranuclear region that exhibit a number of continuities with the outer nuclear membrane. This distribution of e.r. possibly reflects its origin from the nuclear envelope and its participation in parenthesome differentiation.

Fig. 2. A pair of parallel e.r.-elements whose blurred central region may be a very early stage of parenthesome differentiation. 

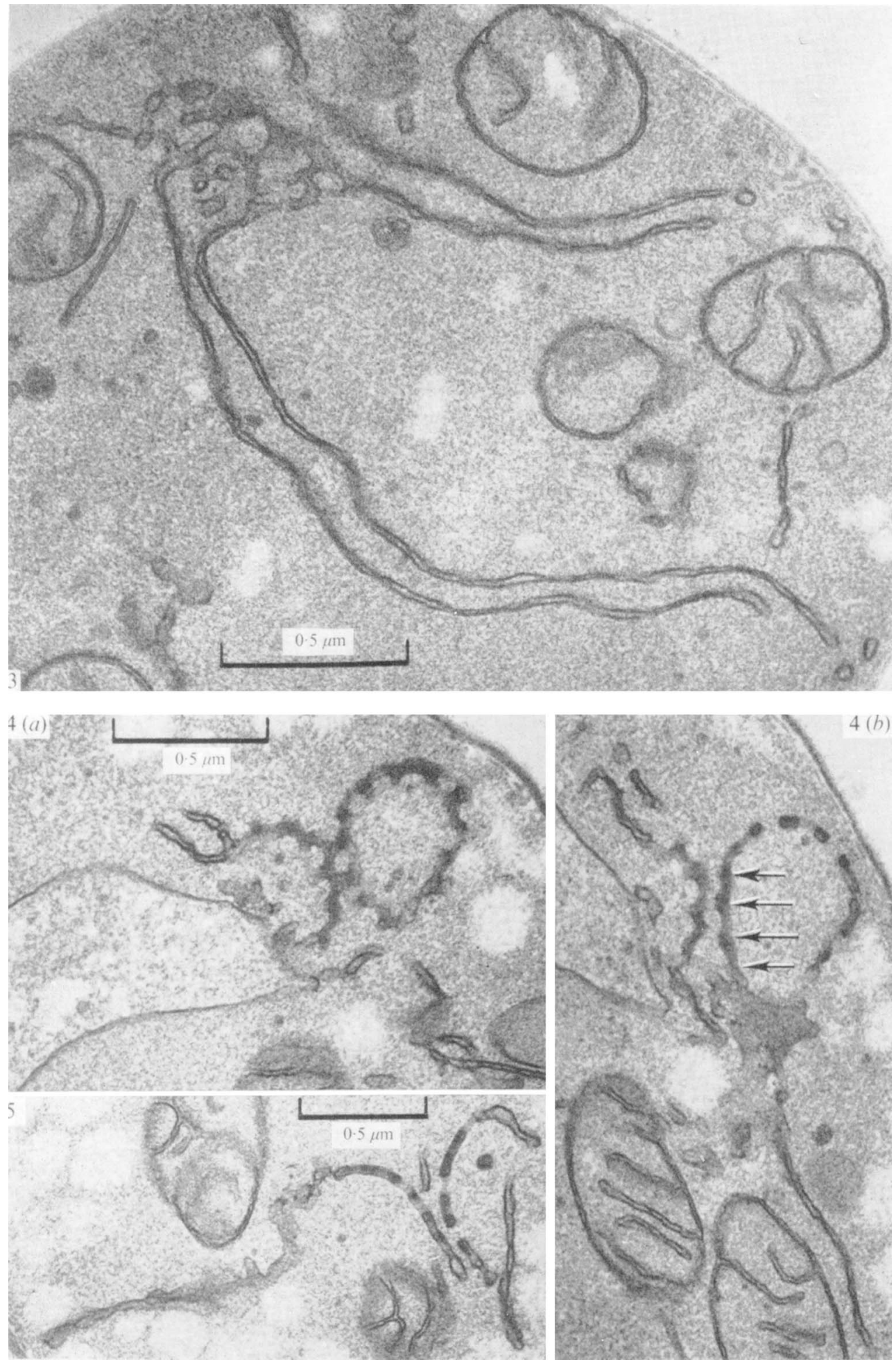

Fig. 3. Later differentiation of paired parallel e.r.-elements in which the central region has become mutually fenestrated.

Fig. 4. Cytoplasmic parenthesomes; $(a)$ and $(b)$ are serial sections of a recently differentiated parenthesome pair that are still partially conjoined. In $(a)$ the contiguity is prominent, while in $(b)$ partial separation has occurred and the bridging connexions (opposite arrows) have become attenuated; e.r. continuities are evident and a portion of the hyphal wall appears in the upper right-hand corner.

Fig. 5. Discrete cytoplasmic parenthesomes; a portion of the hyphal wall appears in the lower lefthand corner. 

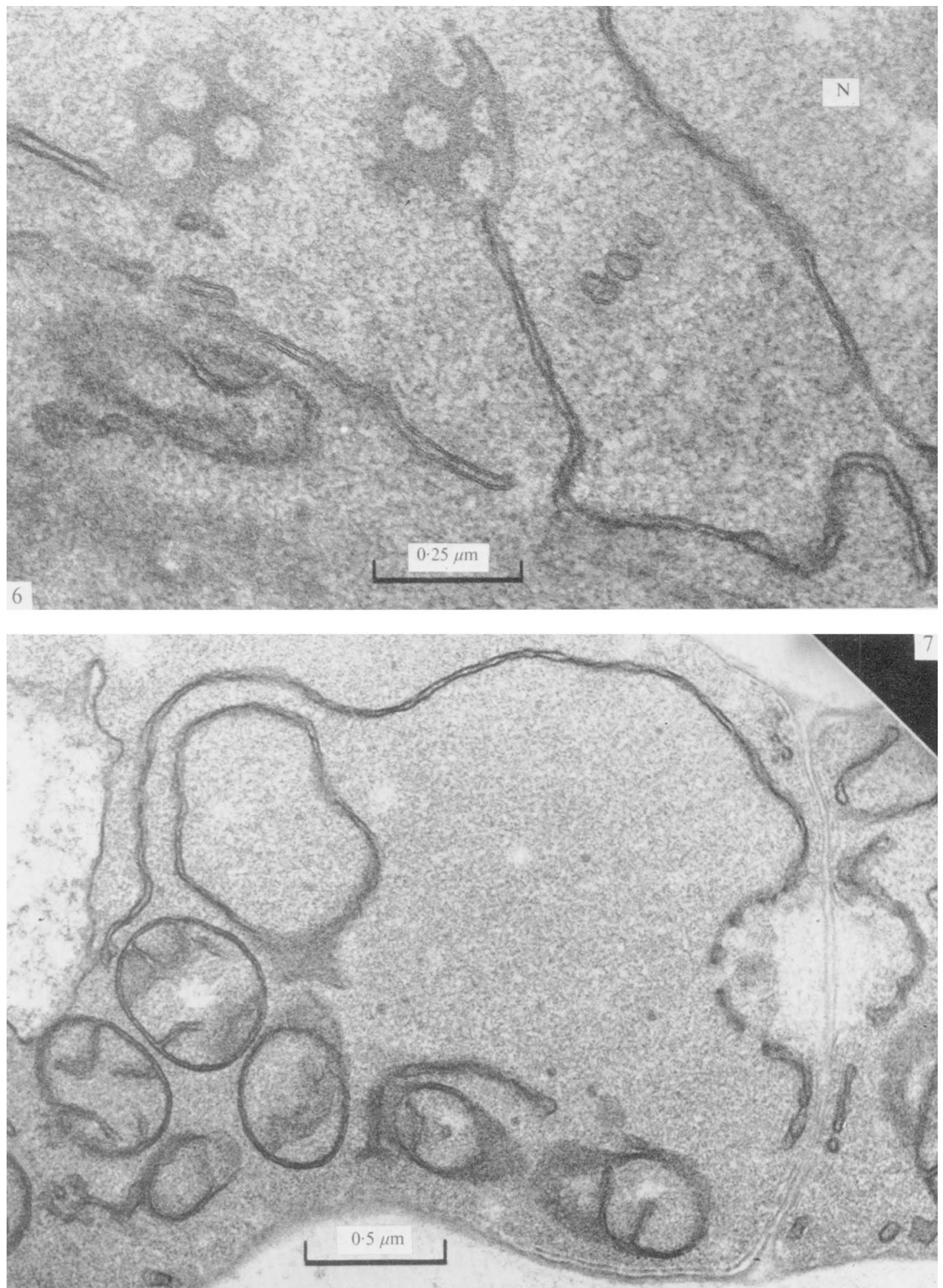

Fig. 6. Two tangential parenthesome sections that may be part of a single parenthesome. The typical pores and the abrupt transition between undifferentiated e.r. and e.r. differentiated into parenthesomes is clearly demonstrated. A portion of a nucleus $(\mathrm{N})$ is shown.

Fig. 7. Longitudinal section of part of a hyphal cell showing an e.r. continuity between a vacuole and a parenthesome; note the absence of wall e.r. The near median septal section passes tangentially through the dolipore and one occlusion (right) and clearly shows the trilamellate nature of the septum. 

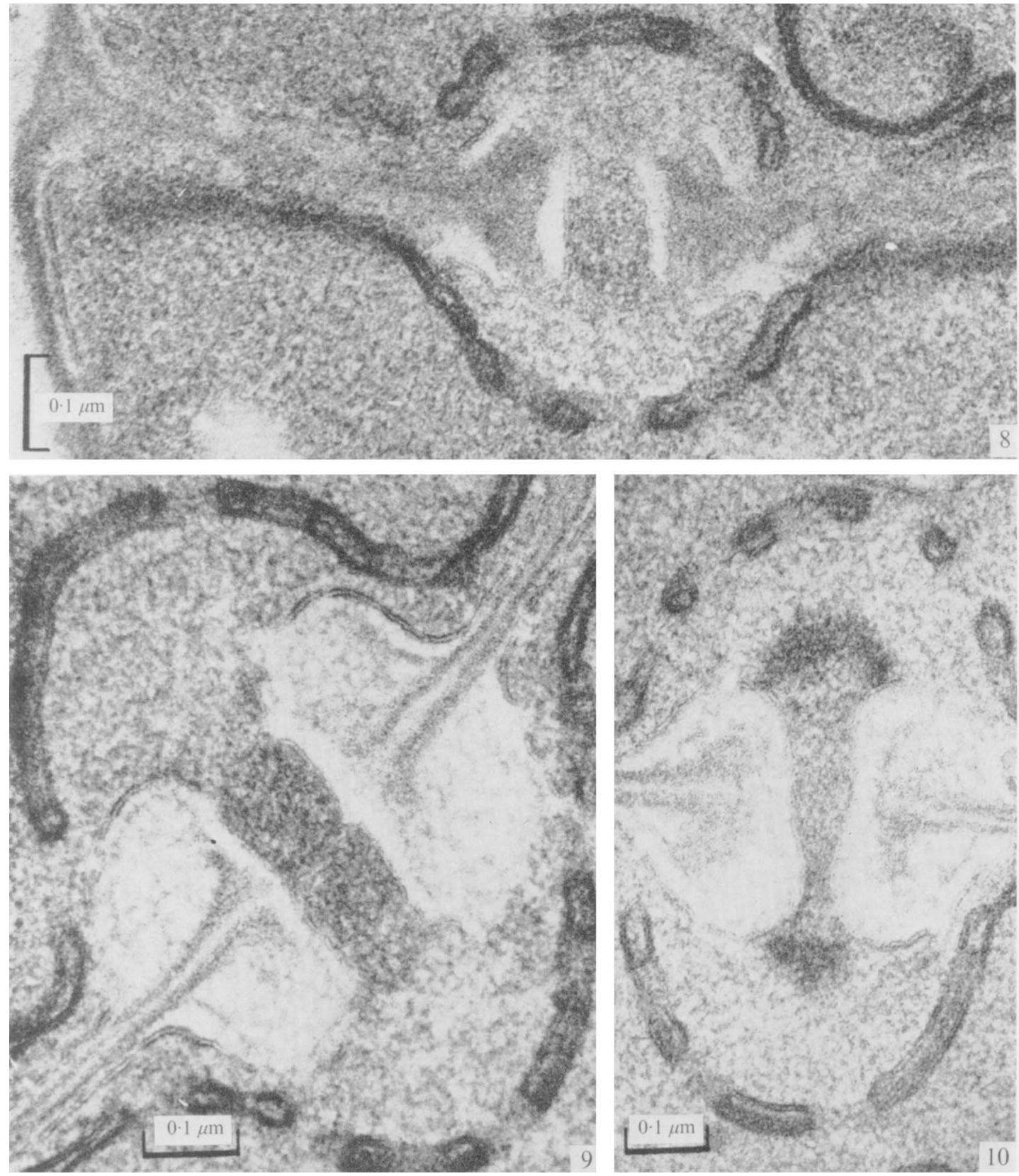

Fig. 8. Immature dolipore septum. The dark material of the dolipore core, continuous with similar material of the peripheral disc, forms a Y-shaped ring within whose angles are three electrontransparent rings that appear in section as light plaques just under the inflated lip of the plasma membrane. The aperture of the dolipore contains the first evidence of a kernel of material whose presence is believed to be responsible for the impeding, flattening, and inflation of the centripetallyclosing membrane margin. (See Fig. I $a$ to $c$ for a diagrammatic interpretation.) The conspicuous parenthesomes are well developed.

Fig. 9. Maturing dolipore. The electron-transparent plaques have expanded and fused to form a complete shell of light material lining the dolipore, the core has expanded and become more diffuse (compare with Fig. 8 at the same magnification), and the apertural kernel has become compact, election dense, and figure-eight-shaped (bipartite). The bilamellate nature of the mature parenthesome is clearly evident. (See Fig. I I $d$ for a diagrammatic interpretation.)

Fig. IO. Mature dolipore septum. It is believed that the compression of the apertural kernel has caused most of the material to be squeezed out to the opposing orifices as dark occlusions, leaving behind traces of material in the channel. (See Fig. I $e$ for a diagrammatic interpretation.) The bilamellate nature of the mature parenthesome is clearly evident. 


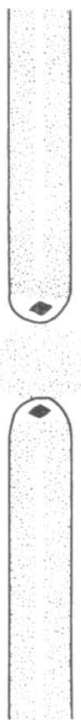

(a)

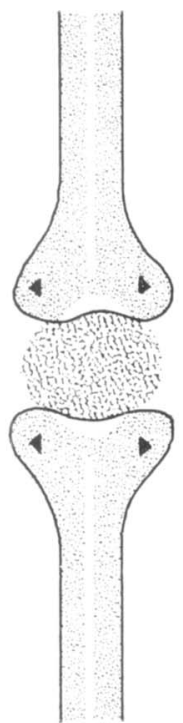

(b)

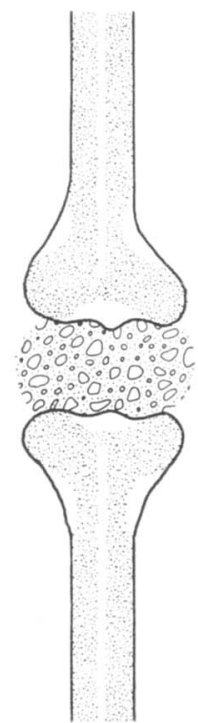

(c)

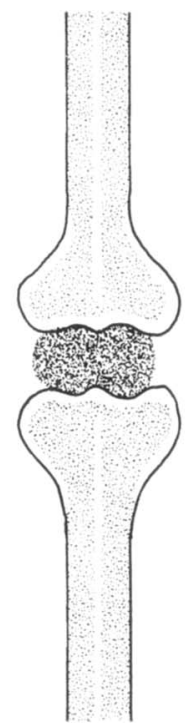

(d)

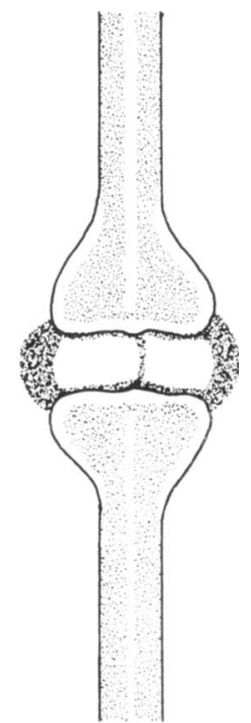

(e)

Fig. I I. Speculative diagram of dolipore formation. (a) Middle portion of the closing septal disc at the instant when the centripetally invaginating lip of the plasma membrane has come against the presumably uncleavable central kernel, which at this stage of development, and through to $(c)$, has the same clarity as the cytoplasm. The trilamellate nature of the septum is indicated, and it is suggested that the electron-transparent central wafer may be generated by a particular portion of the ingrowing margin ( symbol). (b) The still-advancing membrane lip in its final growth has partially grown around the central kernel to form the mould of the dolipore and the bifurcation of the lip has divided the '-region' into two ' -regions'. (c) The three regions producing electrontransparent material have generated three rings of light material that appear in section as plaques in the axes of a dark, roughly $Y$-shaped, presumably ring-shaped structure that is continuous into the outer layers of the disc. The apertural kernel, still dark, has become finely vesicular. $(d)$ Formation of additional electron-transparent material has merged the three previously-discrete regions into a transparent dolipore shell. The apertural kernel has become compressed, electron-dense, and figure-eight-shaped (bipartite). (e) Further dolipore compression has split the apertural kernel and squeezed most of each half out of the opposing orifices. The result is that both moieties are champagne-cork-shaped with the respective 'tops' forming an opaque occlusion at each orifice.

\section{DISCUSSION}

Cell division in the fungi, in contrast to other eukaryotes, is effected by plasma-membrane invagination. In mycelium of the phycomycetes this results in an abscissional cross wall, but in the higher fungi (superdivision Septomycotera; Moore, I97I, I974) the outcome is hyphae with septa that are perforate. In neither the ascomycetes nor the basidiomycetes is the development of septa well understood. In the ascomycetes the material of the septum has approximately the same electron-light appearance as the wall of the hypha and the large, simple pore is guarded by Woronin bodies - generally one on each side, but additional ones may also be present in the pore region. It has been suggested that in these fungi the pore may be formed by the dissolution of a diaphragm composed of the medially-fused opposed folds of the centripetally-invaginated plasma membrane (Moore, 1965) and that the Woronin bodies are in some way tethered (McKeen, 1971) and formed from microbodies (Wergin, 1973).

In the basidiomycetes, however, the evidence is that membrane invagination does not go to completion. I propose that the shape of the dolipore results from the presence of a central, 
dense kernel of protoplasm that impedes the centripetally closing membrane front and causes it, before it stops its inward advance, to flow around an apparently uncleavable kernel to establish the mould of the future dolipore (Fig. I I). Although this invaginating cytokinetic membrane is an infolded extension of the hyphal plasma membrane, the wall it deposits is not equivalent, morphologically or biochemically, to the lateral wall to which it is attached. As in the ascomycetes, the septum is produced by wall material being deposited between two membranes rather than by a single membrane secreting wall substances on to the cell surface (most actively the hyphal tips). What is the site(s) of synthesis of the septal material? The most active region of the septal membrane is the inward growing rim. Although the motive force for invagination is unknown, there is no reason, on first consideration, to expect the centripetal membrane front to produce a different kind of wall material from that produced by the plasma membrane at the hyphal tip. As noted above, ascomycete septa resemble the lateral wall; the respective compositions of these hyphal components, though, is unknown. In the basidiomycetes, however, the septum is composed of three layers: a central light layer, bounded on either side by dark ones (Bracker \& Butler, 1963; Kreger-van Rij \& Veenhuis, 197I) (Fig. 7). How could this configuration arise? Again, it would be expected that the inward closing membrane margin would be the most active site of synthesis. However, is it now differentiated into three zones that are simultaneously secreting two types of material, or are the dark outer layers being formed by the mature or maturing membrane away from the centripetal front? Information that would support one of these hypotheses, or an alternative hypothesis, is not available. The one study that is available, on the septa of Schizophyllum commune (Wessels \& Marchant, 1974), shows that the septal disc contains chitin covered by alkali-insoluble R-glucan and that the dolipores are composed of an alkali-soluble component that is not S-glucan, although this substance is prominent in the lateral walls. The finding that the dolipores in $S$. commune are of a different composition from the septal disc complements the observations here in $P$. biennis. That is, in sections of young septa (Fig. 8) plaques of light material are observed on the face and shoulders of the developing dolipore. The stereo-configuration of the immature dolipore at this stage is thus roughly that of a flanged ring, $Y$-shaped in section, of dark material whose angles are occupied by rings of light material. In some manner, additional deposition of electron-transparent material completes the dolipore shell (Figs. 9 and Io).

The apertural kernel has received little attention. Its existence was first intimated by Moore (1965), but has not been discussed in any of the subsequent papers on basidiomycete septal development. Electron micrographs (Figs. 8 to 10) and the interpretive diagram (Fig. I I) suggest how it may be transformed into the paired occlusions. In Fig. 8 there is the first suggestion of finely vesiculate cytoplasm within the dolipore (Fig. I I $a$ to $c$ ); by Fig. 9 this material has become, perhaps by compression from the maturing dolipore, electron-dense and figure-eight-shaped (i.e. bipartite) (Fig. I $d$ ). Maturation of the dolipore and additional compression of the figure-eight-shaped kernel causes the paired moieties to split and to be squeezed out to the respective dolipore orifices, leaving behind a 'skin' of translucent materials (Figs. Io and I $e$ ). This last step produces the champagne-cork-shaped occlusion (a dark cap subtended by a slight residual shell) described by Moore \& Marchant (1972). Inasmuch as the immature apertural kernel is virtually indistinguishable from the surrounding cytoplasm, its early presence and ontogeny will be difficult to discern. If the blockedcytokinesis model of septum formation is valid then it might be expected that the absence of an apertural kernel would allow complete cell division. Such may be the case in the partition septa of the basidia of the Heterobasidiomycetes and also, perhaps, in basidiospores and other spores of these fungi. 
The parenthesomes are the other major component of the basidiomycete septum. It has been evident since their earliest description that they are differentiated from the e.r. (see Moore, 1965; Bracker, 1967) (Fig. 6), but piecing together the ontogeny of this differentiation has proved difficult. From the evidence reported here it would appear that the sequence in $P$. biennis starts with the production of additional e.r. from the nuclear envelope, followed by the association of e.r. elements in pairs, and the differentiation within these of fenestrations that are the anlage of the pores. The pore-field segments of the e.r. elements subsequently mature into paired parenthesomes that are free in the cytoplasm. Still unresolved is the sequence that brings parenthesomes and dolipore together into the final dolipore/ parenthesome complex.

Part of this work was done in the Laboratory of Ultrastructural Biology, The University of Groningen, Haren, The Netherlands. Grateful acknowledgement is made to them for use of facilities, to Professor J. G. H. Wessels for his hospitality, and to the Nederlandse Organisatie voor Zuiver Wetenschappelijk (ZWO) for their financial support. I also thank Shirley Keeley for preparing the diagram.

\section{REFERENCES}

Bracker, C. E. (1967). Ultrastructure of fungi. Annual Review of Phytopathology 5, 343-374.

BraCKer, C. E. \& BUTLER, E. E. (I963). The ultrastructure and development of septa in hyphae of Rhizoctonia solani. Mycologia 55, 35-58.

GirbardT, M. (I96I). Licht- und elektronenmikroskopische Untersuchungen an Polystictus versicolor. II. Die Feinstruktur von Grundplasma und Mitochondrien. Archiv für Mikrobiologie 39, 35I-359.

Jersild, R., Mishkin, S. \& Niederpruem, J. (1967). Origin and ultrastructure of complex septa in Schizophyllum commune development. Archiv für Mikrobiologie 57, 20-32.

KREGER-VAN RIJ, N. J. W. \& VeEnhUIS, M. (197I). A comparative study of the cell wall structure of basidiomycetous and related yeasts. Journal of General Microbiology 68, 87-95.

MCKEEN, W. E. (I97I). Woronin bodies in Erysiphe graminis DC. Canadian Journal of Microbiology 17, I $557-1560$.

Moore, R. T. (1965). The ultrastructure of fungal cells. In The Fungi, an Advanced Treatise, vol. I, pp. 95I 8 . Edited by G. C. Ainsworth and A. S. Sussman. New York and London: Academic Press.

Moore, R. T. (I97I). An alternative concept of the fungi based on their ultrastructure. In Recent Advances in Microbiology (Proceedings $\mathrm{X}$ International Congress for Microbiology). Edited by A. PerezMiravette and D. Pelaez. Mexico, D. F.: Libreria Internacional, S. A.

Moore, R. T. (1974). Proposal for the recognition of super ranks. Taxon 23, 650-652.

MoORE, R. T. \& MARChANT, R. (1972). Ultrastructural characterization of the basidiomycete septum of Polyporus biennis. Canadian Journal of Botany 50, 2463-2469.

RAUDASKoski, M. (1970). Occurrence of microtubules and microfilaments, and origin of septa in dikaryotic hyphae of Schizophyllum commune. Protoplasma 7o, 4I 5-422.

Wergin, W. P. (1973). Development of Woronin bodies from microbodies in Fusarium oxysporum f. sp. lycopersici. Protoplasma 76, 249-260.

Wessels, J. G. H. \& MARChant, R. (I974). Enzymic degradation of septa in hyphal wall preparations from a monokaryon and a dikaryon of Schizophyllum commune. Journal of General Microbiology 83, 359-368. 